\title{
Design of Curvilinear Array Apertures for 3D Ultrasonic Imaging
}

\author{
Oscar Martínez-Graullera ${ }^{1}$, Luis Gomez-Ullate², David Romero ${ }^{3}$, Carlos J. \\ Martín ${ }^{4}$ and Gregorio Godoy ${ }^{5}$ \\ ${ }^{1,2,3,4}$ Centro de Acústica Aplicada y Evaluación No Destructiva. UPM-CSIC. Ctra. Campo \\ Real KM 0,200 28500 Arganda del Rey (Madrid) \\ ${ }^{5}$ Dpto. Ing Electrónica. Universidad de Jaen \\ Spain
}

\section{Introduction}

The development of ultrasonic volumetric imaging is closely linked to the development of systems that are able to operate bidimensional array transducers. These arrays are useful for ultrasonic volumetric imaging, because they produce steered and focused beams throughout a volume of interest. Typical 2-D arrays are based on a Squared Matrix (SM) configuration, where the array elements are the matrix cells. Their performance is determined by their width in terms of wavelenght. Resolution and the dynamic range are determined by wavelenght/aperture diameter ratio and number of elements and the wavelenght/ interelement distance ratio respectively (Smith et al. (1991)).

In SM apertures, since element distribution is uniform, the composition of the secondary lobes is determined by interelement distance. All elements contribute to its formation. These lobes are known as grating lobes and produce image artifacts that can reduce the signal-to-noise ratio. Nowadays avoiding image artifacts is key to array design. For matrix and linear arrays the composition of these lobes can only be avoided by limiting the interelement distance to $\lambda / 2$. In practice, it means that for $1^{\circ}$ of lateral resolution a $60 \lambda$ diameter aperture with 14400 elements is needed. Then, several problems can be identified:

- Thousands of electronic channels are needed, which increases the cost and complexity of the imaging system.

- Thousands of elements are needed, which increases the complexity of the transducer manufacture.

- The small size of the elements is associated to low signal-to-noise ratios.

- Considerable difficulties in making the electrical connections.

These problems pose a technological challenge which is the basis for the main research lines in array design. To meet these challenges and reduce the cost and complexity of 3-D systems, the main design strategy is to break the periodicity in the aperture, using different thinning strategies to reduce the number of active elements and maintaining good field characteristics at the same time(Smith et al. (1991); Schwartz \& Steinberg (1998)). 
Traditionally, the design strategy for matrix distribution has been based on undersampling the 2-D array by connecting only some of the available elements (Hendricks (1991)). Array designers then try to select those elements that produce the most appropriate beam pattern or image for a given set of constraints (Smith et al. (1991); Hendricks (1991); Schwartz \& Steinberg (1998); Lockwood \& Foster (1996)). Most thinning solutions are based on random distribution but over the last decades some solutions that use the multiplicative nature of the pulse-echo process have been presented (Schwartz \& Steinberg (1998); Lockwood \& Foster (1996); Austeng \& Holm (2002); Nikolov \& Jensen (2000)). It has been proposed that an imaging system can be composed by two different apertures in emission and reception, where the grating lobes in emission and the grating lobes in reception appear at different positions and thus they are partly suppressed in the two-way response. However, these solutions are mainly aimed at reducing the number of elements; elements are still very small. For such solutions the SNR can become too low and in general the image contrast achieved is only limited.

Alternatively apertures with curvilinear deployment have been proposed (Schwartz \& Steinberg (1998); Mendelsohn \& Wiener-Avnear (2002); Ullate et al. (2006); Bavaro et al. (2008); Sumanaweera et al (1999)). Unlike matrix distributions, curvilinear apertures have no privileged direction. Therefore only some and not all elements generate grating lobes and thus curvilinear apertures outperform rectilinear designs. Furthermore, curvilinear arrays can be designed so that grating lobes are distributed in wide areas, significantly reducing their impact. Therefore, layouts with inter-element distance beyond $\lambda / 2$ can be considered. In this sense, in curvilinear arrays the number of elements is reduced while element size can be increased which improves the signal-to-noise ratio associated with element impedance and the array active area (Ullate et al. (2006); Martínez-Graullera et al (2010)).

In this paper, two curvilinear designs are analysed. On the one hand, array designs based on the Fermat Spiral (FS), an alternative that provides both curvilinear deployment and reduction in the number of elements. On the other hand, Segmented Annular Apertures or Circular Apertures (CA) which is a curvilinear array composed of a set of concentric rings that are divided into sectors. Grating lobes are not eliminated, but they are spread over a large region generating a pedestal sidelobe. It is shown that the formation of grating lobes can be modelled with a reduced number of parameters. The design parameters of both apertures are studied, and specific thinning strategies are presented.

Some issues have been considered for greater focus on specific problems:

1. Taking into account the technology nowadays available, the number of elements in the aperture is limited to 128 .

2. The configuration considered is composed by different apertures in emission and reception.

3. The apertures have a diameter of $60 \lambda$, providing a lateral resolution of $1^{o}$.

4. The minimum inter-element distance is $\lambda$ so that element area can be increased up to that size.

\section{Analysis tools}

Three different mathematical models are used for analysis and design strategies.

- Narrowband analysis. It models the response at a given frequency, producing the worst case of interference. Therefore, it can be used as a reference for performance asessment. 
This model allows to identify which elements determine the formation of grating lobes. The knowledge adquired from this analysis can be used to develop a desing strategy. From the computational point of view, it is the fastest simulation model, adequate for intensive computation operations.

- Wideband analysis. It is based on point elements and the spatial impulse response computational model. Its computational cost is higher than that of the narrowband, but it provides a good estimate of aperture behaviour without interference from elements response. A design strategy, based on the parameters identified in the narrowband analysis, can be developed using optimization algorithms.

- Wideband acoustic field modelling. The model presented here is based on the spatial impulse response and the Piwackovski solution (Piwakowsky \& Sbai (1999)). It is used to validate the best solution obtained .

Each model can be used at different design stages. It is a three-part process: learning (Narrowband and Wideband analysys), searching (Wideband analysys), and evaluating (Wideband acoustic field modelling). All three stages compose the general procedure for array design.

\subsection{Narrowband analysis tool}

The first stage of the design process (Learning), in which an array model of punctual elements, narrowband excitation and far field approximation are used, is based on the Array Factor response (AF). The Array Factor is a very fast computational model that explains or shows how the difraction pattern is developed for different aperture parameters. In this way, it helps us identify which are the key parameters and design the most appropriated strategy to improve the array performance.

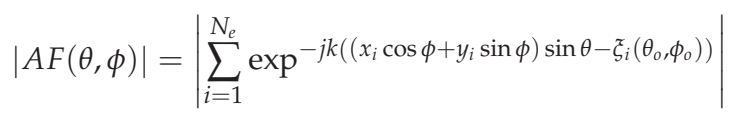

Where $\left(x_{i}, y_{i}\right)$ are the cartesian coordinates of the $i^{\text {th }}$ element and $\xi_{i}\left(\theta_{0}, \phi_{0}\right)$ is the corresponding phase delay value to focus on $\left(\theta_{0}, \alpha_{0}\right)$.

$$
\xi_{i}\left(\theta_{0}, \phi_{o}\right)=\left(x_{i} \cos \phi_{o}+y_{i} \sin \phi_{o}\right) \sin \theta_{o}
$$

The Array Factor response in pulse-echo can be evaluated using:

$$
\left|A F_{e r}(\theta, \phi)\right|=\left|A F_{e}(\theta, \phi) A F_{r}(\theta, \phi)\right|
$$

Where $A F_{e}(\theta, \phi)$ and $A F_{r}(\theta, \phi)$ are respectively the array factor on emission and reception of aperture, which are computed by equation 1 .

\subsection{Wideband analysis tool}

A wideband model is based on the spatial impulse response. Punctual elements are used. Echo-pulse response can be modelled by Nikolov \& Jensen (2000):

$$
s(\vec{x}, t)=\frac{1}{c^{2}} \frac{\partial^{2} v(t)}{\partial t^{2}} *\left\{h_{T}(\vec{x}, t) * h_{R}(\vec{x}, t)\right\}
$$

Where $h_{T}(\vec{x}, t)$ and $h_{R}(\vec{x}, t)$ are the emitting and the receiving spatial impulse response of the array, and $v(t)$ is the excitation signal. To reduce the computational cost and suppress the 
element diffraction response, each one is reduced to a pointwise located at the center of the element. Then, the spatial impulse response of the array is computed as:

$$
h(\vec{x}, t)=\sum_{i=1}^{N_{e}} \frac{\delta\left(t-\frac{r_{i}}{c}-T_{i}\right)}{2 \pi r_{i}}
$$

Where $r_{i}$ is the distance from the center of the $i^{\text {th }}$ element to the field point $\vec{x}$, and $T_{i}$ is the corresponding focussing delay. Then, using equation 5 to model emission and reception arrays in equation 4 , the presure wave obtained is mainly determined by excitation pulse and element distribution in the aperture.

The Point Spread Function (PSF) in wide-band in a $\vec{x}$ position can be calculated by the maximum value of the modulus of $s(\vec{x}, t)$. The PSF is evaluated in a hemisphere as follows:

$$
\operatorname{PSF}(\theta, \phi)=\max _{t}\left(\left|s\left(\left(R=\frac{D^{2}}{(4 \lambda)}, \theta, \phi\right), t\right)\right|\right)
$$

Futhermore, the equation 4 is used to obtain the general expression of the acoustic pressure generated by an array working in emission-reception. Where $h_{E}$ and $h_{R}$ are, respectively, the impulse responses in emission and reception of the array obtained by the sum of the spatial impulse response of each element:

$$
\begin{aligned}
& h_{E}(\vec{x}, t)=\sum_{i=1}^{N_{e}} a_{i}^{E} h_{i}\left(\vec{x}, t-T_{i}^{E}\right) \\
& h_{R}(\vec{x}, t)=\sum_{i=1}^{N_{e}} a_{i}^{R} h_{i}\left(\vec{x}, t-T_{i}^{R}\right)
\end{aligned}
$$

Where $T_{i}^{E}$ and $T_{i}^{R}$ are, respectively, the focusing delays in emission and reception, $a_{i}$ is its gain, and $h_{i}$ is the impulse response of the $i^{\text {th }}$ array element computed by the Rayleigh integral.

$$
h_{i}(\vec{x}, t)=\iint_{A_{i}} \frac{\delta\left(t-\frac{r_{i}}{c}-T_{i}\right)}{2 \pi r_{i}} \delta s
$$

Where $A_{i}$ is the area of the emitting/receiving transducer. The Piwakowsky method was used to calculate this equation. This method makes a direct computation of the Rayleigh integral by means of transducer surface discretization into squared cells of elemental area $\Delta S$, and time sampling with intervals of $\Delta t$. Hence, the contribution of every element to the array impulse response at a given instant $t_{S}$ is obtained by adding the contributions of the cells contained between two concentric spherical waves, which are separated by the discretization interval $c \Delta t$ (Piwakowsky \& Sbai (1999)). Thus, the contribution of the $i^{\text {th }}$ array element at the instant $t=t_{s}$ is given by:

$$
\begin{gathered}
h_{i}\left(t=t_{S}\right)=\frac{1}{\Delta t} \sum_{j} b_{j}, t_{S}-\frac{\Delta t}{2} \leq t_{j} \leq t_{S}+\frac{\Delta t}{2} \\
b_{j}=\frac{a_{i} \Delta S}{2 \pi\left(R_{j}-T_{i}\right)} \quad \text { and } \quad R_{j}=c t_{j}
\end{gathered}
$$

Where $T_{i}$ denotes the focusing delay in emission or reception and $R_{j}$ is the distance from each cell to the field point $P(\vec{x})$. 


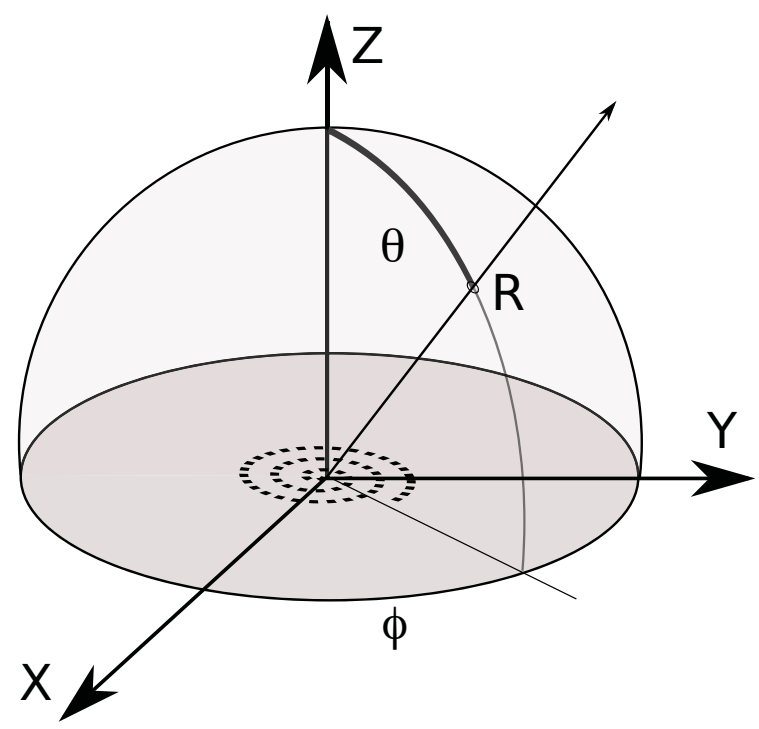

Fig. 1. Coordinate system used to compute de array beampattern. The array is located in the $\mathrm{XY}$ plane $(\theta=0)$ and the field is computed in a hemisphere, defined for narrowband by $(\theta, \phi)$ and for wideband by $(R, \theta, \phi)$.

\subsection{Nomenclature}

A generalized nomenclature is used for the apetures in the design:

$$
F\left(N_{t}, N_{r}\right)
$$

Where $\mathrm{F}$ is the main geometry organization in the element distribution. SP stands for spiral configuration, CA for Circular Apertures and $\mathrm{M}$ for Matrix configuration. $N_{t}$ and $N_{r}$ are the number of elements in the emision and reception aperture respectively. When the apertures in emission and in reception are the same the notation is $\mathrm{F}\left(N_{e}\right)$.

\subsection{Evaluation parameters}

In figure 1 the coordinate system used to evaluate the diffraction response in the hemisphere is presented. For simplicity, the hemisphere is presented as a disk by its projection over the $\mathrm{XY}$ plane. Several examples can be seen in figure 3.

To evaluate the apertures, the acoustic field (narrowband and wideband) is computed in a far field hemisphere $\left(R=\frac{D^{2}}{(4 \lambda)}, \theta=0^{\circ}: 1^{o}: 90^{\circ}, \phi=0^{\circ}: 1^{\circ}: 360^{\circ}\right)$, focusing on $\left(R=\frac{D^{2}}{(4 \lambda)}, \theta=\right.$ $\left.0^{\circ}, \phi=0^{\circ}\right)$ in emision and reception. Also, lateral profiles in wideband are employed to show the aperture performance. Maximum, mean and minimum sidelobes at each elevation angle $\left(\theta=0^{\circ}: 1: 90^{0}\right)$ are shown. See figure 6 for examples.

With this results, a number of parameters was considered to assess apertures in wideband:

- The lateral resolution at two levels of $-6 \mathrm{~dB}$ and $-40 \mathrm{~dB}$, in order to analyse the main lobe sharpness

- The grating lobe maximum level, which is determined by analysing the PSF for $60 \%$ signal bandwidths: 
- The mainlobe-to-sidelobe energy ratio (MSR).

This last parameter MSR was proposed in Nikolov \& Jensen (2000) as a measurement of how energy is spread in the field, and can be computed by:

$$
M S R=20 \log \frac{\sum \sum|P S F(\theta, \phi)|^{2} M L(\theta, \phi)}{\sum \sum|\operatorname{PSF}(\theta, \phi)|^{2}(1-M L(\theta, \phi))}
$$

Where $\operatorname{ML}(\theta, \phi)$ is a logical function that delimits the main lobe region. Here, due to the nature of the results, we considered that the main lobe is defined by those points having pressure amplitude higher than $-40 \mathrm{~dB}$ :

$$
\operatorname{ML}(\theta, \phi)=\left(20 \log \frac{\operatorname{PSF}(\theta, \phi)}{\operatorname{PSF}(0,0)}>-40 d B\right)
$$

\section{Arrays desing based on fermat spiral}

In the search of a pattern for element distribution with reduced periodicity, we also worked on the Fermat Spiral. Although previous studies looked into the spiral layout (Sumanaweera et al (1999)), the Fermat spiral has not been studied yet. Fermat spiral is defined by the divergence angle $\alpha$ that determines the angular distance between two consecutive elements. The radial position of each element is determined by the square root of its angular position.

$$
x_{n}=\left(R_{n}, \alpha_{i}\right)=\left(R_{0} \sqrt{n \alpha}, n \alpha\right), n=0, \ldots\left(N_{e}-1\right)
$$

Where the element centre $x_{n}$ is defined by its polar coordinates and $N_{e}$ is the number of elements. $R_{0}$ is a constant value which is needed to obtain a particular aperture size. It is defined as:

$$
R_{0}=\frac{D}{2 \sqrt{\left(N_{e}-1\right) \alpha}}
$$

Where $\mathrm{D}$ is the aperture diameter.

In Figure 2 different spiral arrangements with $N_{e}=128$ and divergence angles $\left(\alpha=9^{\circ}, 174^{\circ}\right.$, $116^{\circ}, 92^{\circ}$ ) are shown. It can be seen that although only one line is drawn for the divergence angle, each spiral seems to be configured by different number of branches, or inner spirals, which grow from the centre of the array. We named the configurations of Figure 2 as SPx, where $\mathrm{x}$ is the number of branches.

Figure 3 shows the Array Factor in the plane $\left(\theta=0^{\circ}: 30^{\circ}, \phi=0^{\circ}: 360^{\circ}\right)$ for $\xi_{\theta_{0}, \phi_{0}}=0$ for the apertures shown. The figure shows how grating lobes are organized in branches, generating different diffraction patterns for each divergence angle.

In our opinion, the Fermat spiral is interesting for array design because of a number of issues.

1. Only the divergence angle and the number of elements are needed to define the layout.

2. It is a biological pattern, which in fact it is used by Phyllotaxis as a reference to model several leaves arrangements (Jean (1983); Ridley (1982)).

3. The outer elements show a more compact distribution than other spiral layouts, like Archimedean, Hyperbolic, Lituus or Logarithmic. This compactness leads to the element shadowing effect described in Schwartz \& Steinberg (1998) and Ullate et al. (2006). 


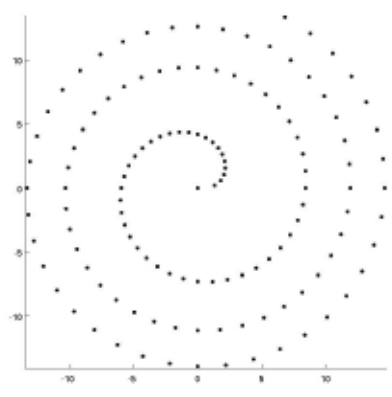

(a) $\alpha=9^{\circ}(\mathrm{SP} 1)$

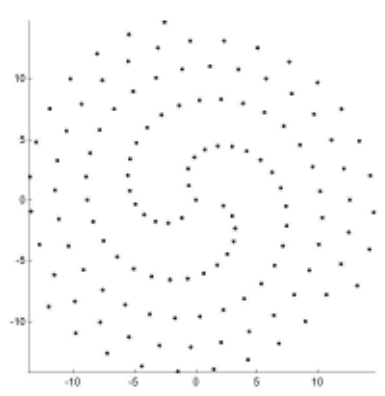

(c) $\alpha=116^{\circ}(\mathrm{SP} 3)$

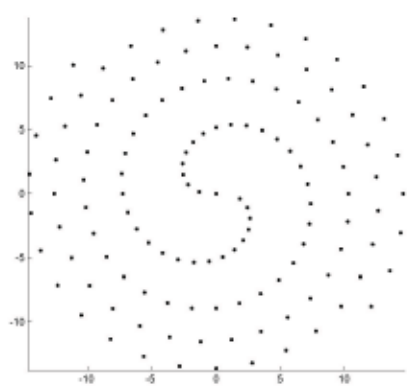

(b) $\alpha=174^{\circ}(\mathrm{SP} 2)$

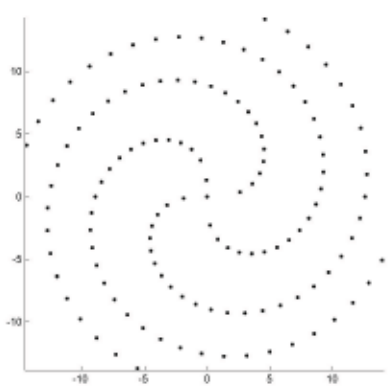

(d) $\alpha=92^{\circ}(\mathrm{SP} 4)$

Fig. 2. Aperture distribution with different spiral arrangements. We have classified them in SP1, SP2, SP3 and SP4, according to the formation of branches.

4. Although elements are organised following a determined line, they compose other spiral structures in the layout. This particular arrangement distributes elements contribution to grating lobes, which are extended over wide but well located areas. Its general level is reduced.

\subsection{Design strategie}

In our project, taking into account the limitations described at the begining of the chapter, we propose a global array composed by two spirals of 128 elements, one for emission and another for reception, composing a configuration SP $(128,128)$. The design strategy is based on the multiplicative nature of the pulse-echo process, and it tries to locate tgrating lobes in emission and grating lobes in reception at different positions. Hence, they are partly suppressed in the two-way response.

This principle can be applied to Fermat layouts where grating lobes are located in well defined positions. Rather than searching different complementary apertures, we use the same aperture for emission and reception and apply a phase displacement $\Delta \alpha$ between them in order to locate emission and reception grating lobes in complementary angular positions. Then the arrays that compose the $\mathrm{SP}(128,128)$ configuration can be determined by the equations: 


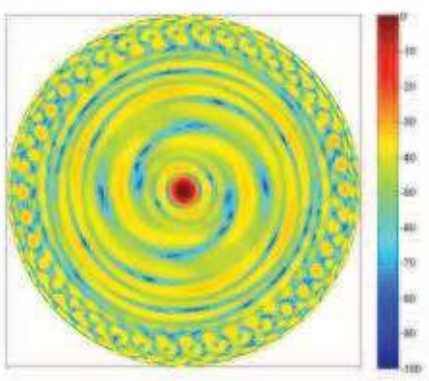

(a) $\alpha=9^{\circ}$ (SP1)

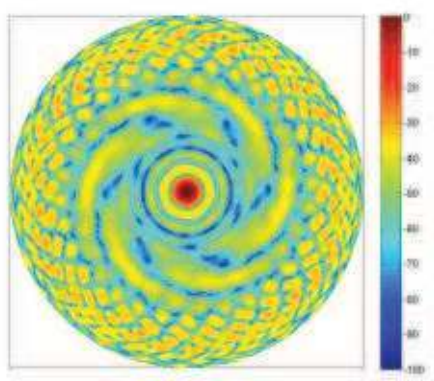

(c) $\alpha=116^{\circ}(\mathrm{SP} 3)$

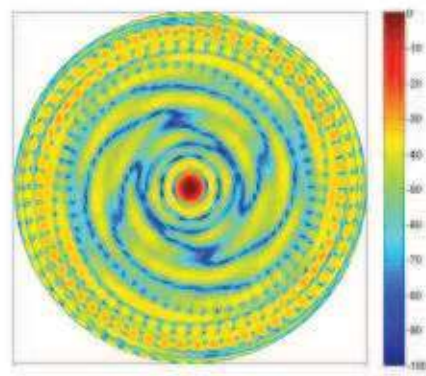

(b) $\alpha=174^{\circ}$ (SP2)

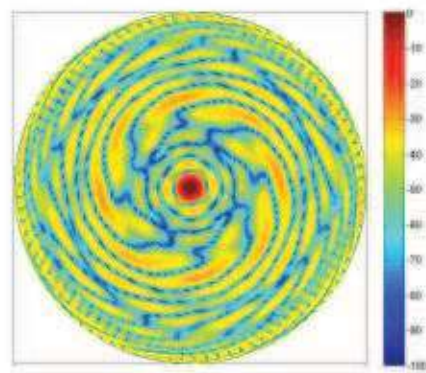

(d) $\alpha=92^{\circ}(\mathrm{SP} 4)$

Fig. 3. Narrowband Array Factor $\theta=0^{\circ}: 30^{\circ}$ for different spiral arragements.

$$
\begin{gathered}
x_{E n}=\left(R_{n}, \alpha_{n}\right)=\left(R_{0} \sqrt{n \alpha}, n \alpha\right), n=0, \ldots 127 \\
x_{R n}=\left(R_{n}, \alpha_{n}\right)=\left(R_{0} \sqrt{n \alpha}, n \alpha+\Delta \alpha\right), n=0, \ldots 127
\end{gathered}
$$

Where, $x_{E n}$ and $x_{R n}$ are the element centres of the emission and reception apertures.

In narrowband, the highly efficient computational model allows an exhaustive analysis. We analysed 18,000 spiral apertures that were obtained varying the divergence angle. For each viable solution with spiral apertures, the two way array factor response of all possible complementary apertures $\left(\Delta \alpha=0^{\circ}: 0.1^{\circ}: 180^{\circ}\right)$ was evaluated.

The grating lobe peak values for all apertures are summarised in figure 4 and table 1 , where the most representative solutions are shown. Although the results for the case $\Delta \alpha=0^{\circ}$ are in the $-15 \mathrm{~dB}$ to $-25.6 \mathrm{~dB}$ range, the combination of two complementary apertures produces a general improvement of the results. The best results are obtained at $-31 \mathrm{~dB}$.

Our best outcome in narrowband shows a grating lobe level of $-43.6 \mathrm{~dB}$ in wideband. In spite of the promising results for narrowband signal, the transferal to wideband (table 1) does not guarantee similar best results. 


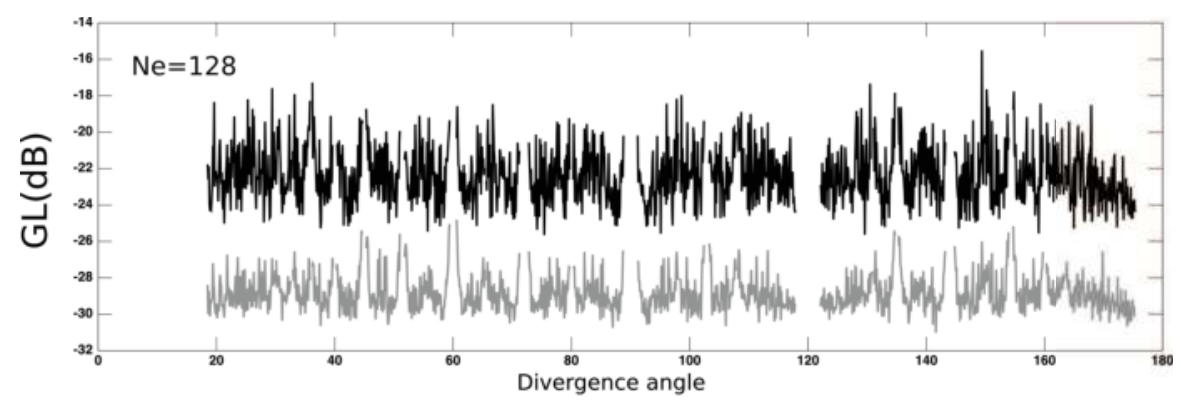

Fig. 4. Narrowband Grating lobe distribution per divergence angle. Black line represents the echo-pulse response if $\Delta \alpha=0^{0}$; the gray line represents the echo-pulse response of the better case obtained when $\Delta \alpha>0^{0}$.

\begin{tabular}{|c|cc|cc|}
\hline & \multicolumn{2}{|c|}{ Narrow-band } & \multicolumn{2}{c|}{ Wide-band } \\
$\alpha$ & $\Delta \alpha=0^{\circ}$ & $\Delta \alpha>0^{\circ}$ & $\Delta \alpha=0^{\circ}$ & $\Delta \alpha>0^{\circ}$ \\
\hline 141.6 & $-23.7 \mathrm{~dB}$ & $-31 \mathrm{~dB}$ & $-40.27 \mathrm{~dB}$ & $-43.61 \mathrm{~dB}$ \\
\hline 75.4 & $\mathbf{- 2 5 . 6 \mathrm { dB }}$ & $-30 \mathrm{~dB}$ & $-37.5 \mathrm{~dB}$ & $-39.9 \mathrm{~dB}$ \\
\hline
\end{tabular}

Table 1. Grating lobe levels for SP $(128,128)$ apertures with best results in narrowband $\left(\Delta \alpha=0^{\circ}\right.$ and $\left.\Delta \alpha>0^{\circ}\right)$; and its corresponding grating lobe levels in wideband. Results for Golden angle are also presented.

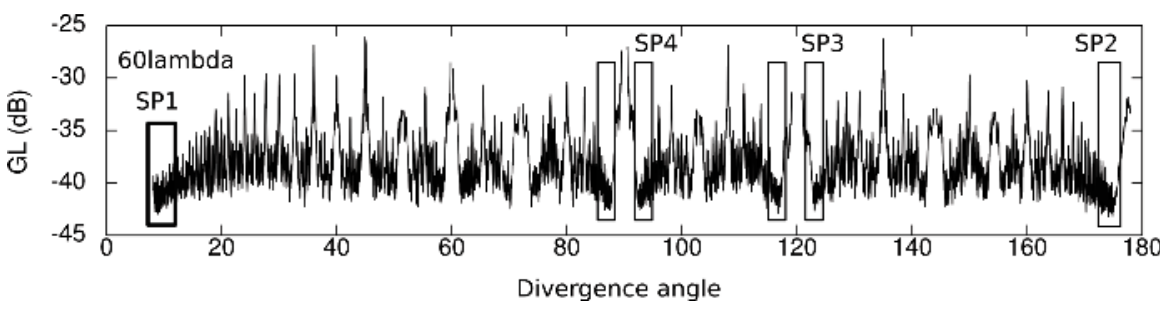

Fig. 5. Grating lobe levels vs the divergence angle, for wideband response (two ways, $\left.\Delta \alpha=0^{\circ}, \lambda=0.5 \mathrm{~mm}, \mathrm{BW}=60 \%\right)$. Rectangles show the location of the best apertures.

\subsubsection{Wideband analysys}

The computational cost of the wideband model is higher than that of the narrowband model. As a consequence it is difficult to produce an exhaustive study. However, from the analysis of narrowband results a reduction in the number of cases can be obtained.

Our First objective is to find the best solution, then with the aim of obtaining the best apertures, an exhaustive search based on the divergence angle is subsequently carried out. Fortunately, the aperture design conditions reduce the total amount of cases under study. Furthermore, the diameter normalisation $R_{N_{e}}=R_{0}$ generates symmetries in the aperture layout that can be used to reduce the total number of apertures and consequently the set of apertures can be reduced to viable solutions of $\alpha$ in $\left(0^{\circ}: 0.005^{\circ}: 180^{\circ}\right)$.

When $\Delta \alpha=0^{o}$ is evaluated for each aperture, it is possible to obtain a general overview of the grating lobe level distribution and thus reduce the set of possible solutions. Results are shown in Figure 5. The three aperture diameters show similar grating lobes distributions but the widest aperture shows more cases, specially for low values of $\alpha$. 
After examining the results, the $-42.5 \mathrm{~dB}$ value was chosen as a grating lobe threshold to limit the set of apertures to be evaluated in the complementary configuration (figure 5). For $\mathrm{D}=60 \lambda, 42$ cases were identified and they could improve the result obtained in the narrowband analysis.

The set of possible solutions is divided in four groups according to element organisation (figure 5) . Figure 2 shows the most representative cases.

- SP1: Aperture defined by one branch. Range of divergence angles $8.5^{\circ}: 9.5^{\circ}$.

- SP2: Aperture defined by two branches. Range of divergence angles $172.8^{\circ}: 175.5^{\circ}$.

- SP3: Aperture defined by three branches. Range of divergence angles $116.5^{\circ}: 123.5^{\circ}$.

- SP3: Aperture defined by three branches. Range of divergence angles $116.5^{\circ}: 123.5^{\circ}$.

- SP4: Aperture defined by four branches. Range of divergence angles $87.5^{\circ}: 93.5^{\circ}$.

\subsubsection{Best solution}

Table 2 shows the best apertures. A viable solution is given in three regions. The number of cases with a grating lobe peak value lower than $-45.5 \mathrm{~dB}\left(N_{a}\right)$, the lateral resolution, the MSR and the grating lobe for $60 \%$ are also shown. No representative cases were found in SP4 region.

\begin{tabular}{|c|c|c||c|c|cc|cc|c|}
\hline Region & $N_{a}$ & best $\alpha$ & $\Delta \alpha$ & $\mathrm{D}$ & \multicolumn{2}{|c|}{ GL Level $(60 \%)$} & \multicolumn{2}{c|}{ Lat. Res. } & MSR \\
\hline SP1 & 7 & $9.3050^{\circ}$ & $66.6^{\circ}$ & $60 \lambda$ & $-46.1 \mathrm{~dB}$ & $-42.5 \mathrm{~dB}$ & $1.0^{\circ}$ & $3.2^{\circ}$ & $17.9 \mathrm{~dB}$ \\
\hline SP2 & 18 & $174.525^{\circ}$ & $46.6^{\circ}$ & $60 \lambda$ & $-46.4 \mathrm{~dB}$ & $-42.5 \mathrm{~dB}$ & $1.0^{\circ}$ & $3.2^{\circ}$ & $18.0 \mathrm{~dB}$ \\
\hline SP3 & 3 & $116.64^{\circ}$ & $29.82^{\circ}$ & $60 \lambda$ & $-45.6 \mathrm{~dB}$ & $-42.5 \mathrm{~dB}$ & $1.0^{\circ}$ & $4.8^{\circ}$ & $17.8 \mathrm{~dB}$ \\
\hline
\end{tabular}

Table 2. Performance of the best $\mathrm{F}(128,128)$ configurations for each region and diameter. Lateral resolution at $-6 \mathrm{~dB}$ and $-40 \mathrm{~dB}$ and grating lobe level. Grating lobe peak levels for pulse bandwidth of $70 \%$ and $80 \%$.

Figure 13 shows the best case $\left(\alpha=174.525^{\circ}\right)$ beampatterns. Lateral profiles show maximum, mean and minimum grating peak lobe level for each elevation angle. Optimised apertures have almost flat profiles with elevation.

\section{Circular aperture}

The Circular aperture, also known as Segmented Annular Aperture, is composed by a set of concentric rings that are divided in several elements. This aperture introduces several design factors that are useful to reduce distribution regularity. For instance, it is possible to use non equal spacing between rings or to use different interelement distance in each ring. Therefore the analysis could be very complex. To reduce the number of variables, this study is focused on what we have called Regular Distribution. This set of apertures is defined by one condition: all elements are the same size and the interelement distance is uniform. With this simple condition two consequences can be formulated:

- The distance between rings is constant.

- The number of elements in each ring is increased with the radius maintaning a similar distance between elements in all rings.

Figure 7 shows an example composed by four rings and the principal design paramenters:

- The radial distance $\left(d_{R}\right)$. 


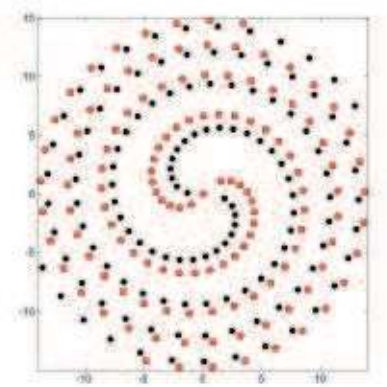

(a) Element distribution: in black emission aperture; in red reception aperture

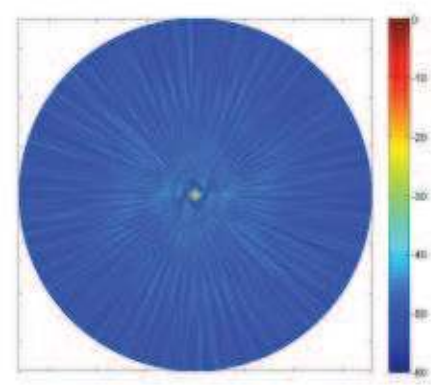

(b) Beampattern in the semisphere

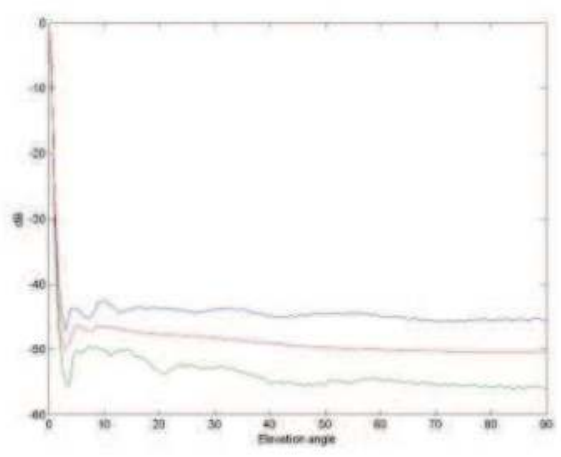

(c) Lateral profile. Maximum, mean and minimum side lobes in elevation are presented

Fig. 6. Best result for $\mathrm{SP}(28,128)$ is presented, $\alpha=174.525^{\circ}$.

- The angular distance $\left(d_{A}\right)$.

- The phase alignment per ring $\left(\phi_{i}\right)$.

- The aperture size $(D)$.

\subsection{Grating lobes distribution}

It is well known that the factor which determines the generation of grating lobes for any array is the distance between elements. The same applies to annular segmented apertures. In this sense the most relevant distances in the aperture are angular and radial distances.

Two apertures were developed to evaluate how these distances determine the grating lobe formation. The aperture shown in figure 8 (a), was designed to garantee that $d_{A}$ is lower than $\lambda / 2$ and $d_{R}=2 \lambda$. The aperture shown in figure $8(\mathrm{~b})$, was designed to garantee that $d_{R}$ is lower than $\lambda / 2$ and $d_{A}=2 \lambda$.

By manipulating these distances we conclude that two different kinds of grating lobes can be easily distinguished: 


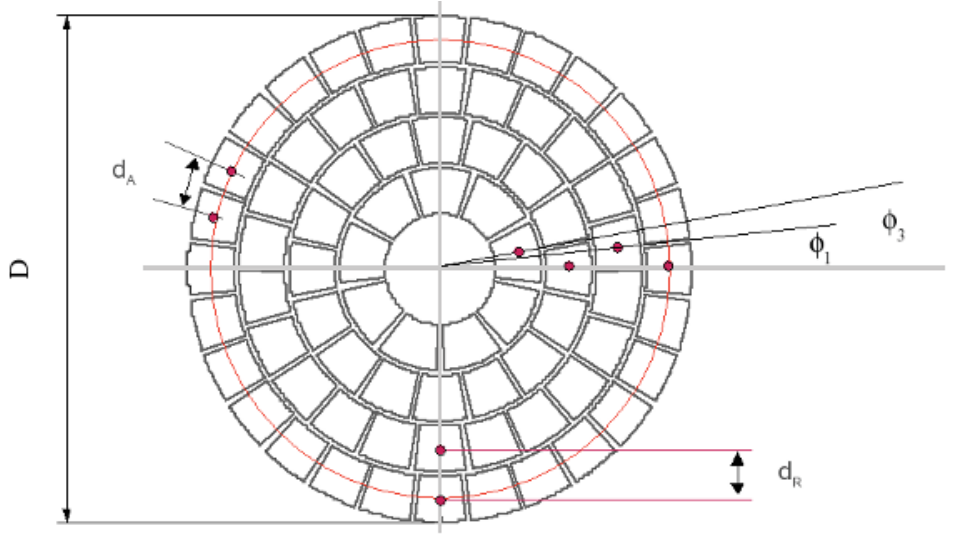

Fig. 7. Annular segmented array.
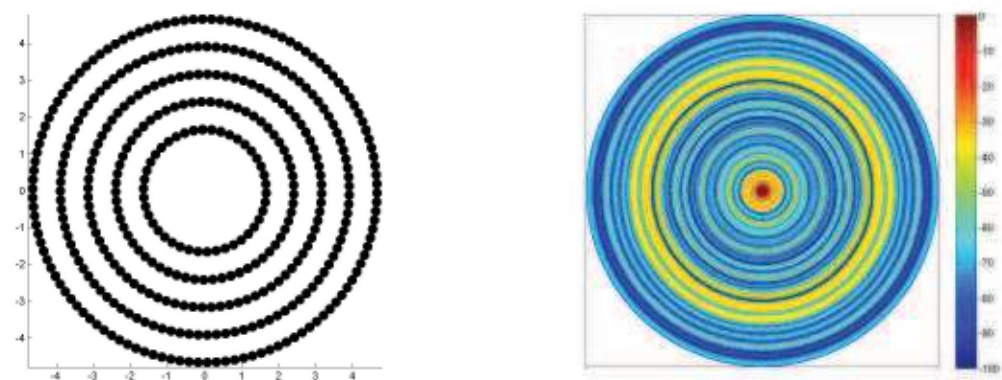

(a) Grating lobes generated by radial distance. Aperture with $d_{A}$ is lower than $\lambda / 2$ and $d_{R}=2 \lambda$.
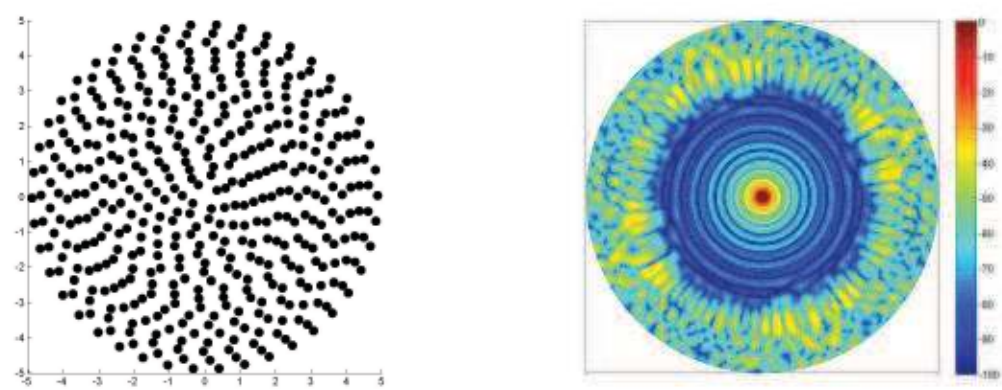

(b) Grating lobes generated by angular distance. Aperture with $d_{R}$ is lower than $\lambda / 2$ and $d_{A}=$ $2 \lambda$.

Fig. 8. Grating Lobe generation in Circular Apertures and main distances between elements. 
- The Radial Lobe $\left(R_{L}\right)$ that has a narrow circular shape and shows continuity for any azimuth direction (figure 8 (a)). Its position is determined by $d_{R}$.

- The Angular Lobes $\left(A_{L}\right)$, beam shaped, are distributed in different elevation positions for each azimuth direction (figure 8 (b)). The nearest lobes are determined by $d_{A}$ distance and the rest are determined by the projection of $d_{A}$ in the linear equivalent array, which depends on the azimuth angle.

Then, the position of this grating lobes can be determined in elevation by the equations:

$$
\begin{aligned}
& R_{L}=\arcsin \left(\frac{\lambda}{d_{r}}\right) \\
& A_{L}=\arcsin \left(\frac{\lambda}{d_{a}}\right)
\end{aligned}
$$

Nevertheless, for a given azimuth direction, the lobes amplitude is linked with the number of coincident elements for that direction, which is not always the same. In the equivalent linear array, our first option to reduce the number of coincidences is changing the element Aspect Ratio (AR), which is the ratio between angular distance $d_{A}$ and radial distance $d_{R}$. The objective of this technique is to avoid the grating lobe reinforcement that occurs when both dimensions are equal.

Figure 9 presents the array factor for three different cases, Aspect Ratio: 0.5, 1.0 and 1.5. All the arrays were designed as demonstrators, with $d_{R}=2 \lambda$ and $D=20 \lambda$, and AF was computed to show how grating lobes can be combined.

- For $\mathrm{AR}=0.5$, both lobes are completely uncoupled and it is easy to remark its properties (figure 9(a)). The Radial Lobe has a narrow circular shape; Angular Lobes have a straight shape and are distributed in different azimuth directions.

- For $\mathrm{AR}=1$, both lobes coincide in the same position and there is a reinforcement, specially for those directions with higher angular lobes (figure 9(b)).

- For $\mathrm{AR}=1.5$, due to the fact that Angular Lobes spread their influence over a wide region there is still a coincidence in position with the Radial Lobes, but with lower intensity than in the $A R=1$ case (figure 9(c)).

Aspect Ratio and phases alignment are both design elements. Phases alligment allows to distribute angular grating lobes by generating an interference pattern between them that can be used to decrease its presence. Phase alignment is implemented by shifting elements from one ring to another in order to avoid privileged directions in the aperture. Phases alignment introduces a random factor in the array design. Figure 10 shows the histogram of the maximum grating lobe obtained in pulse-echo wideband response $(60 \%)$. One thousand random phase-alignment cases for the same aperture with different aspect ratio were computed. The worst results are obtained for $A R=0.5$ where the coincidences in grating lobe position are higher. For $A R=1$ grating lobes values vary between $-29 \mathrm{~dB}$ and $-36 \mathrm{~dB}$, and for $A R=1.5$ the results are quite similar $(-30 \mathrm{~dB}$ and $-37 \mathrm{~dB})$. In short, the figure shows that small corrections in elements positions can improve results by $5 \mathrm{~dB}$, which should be taken into account in the design process. 

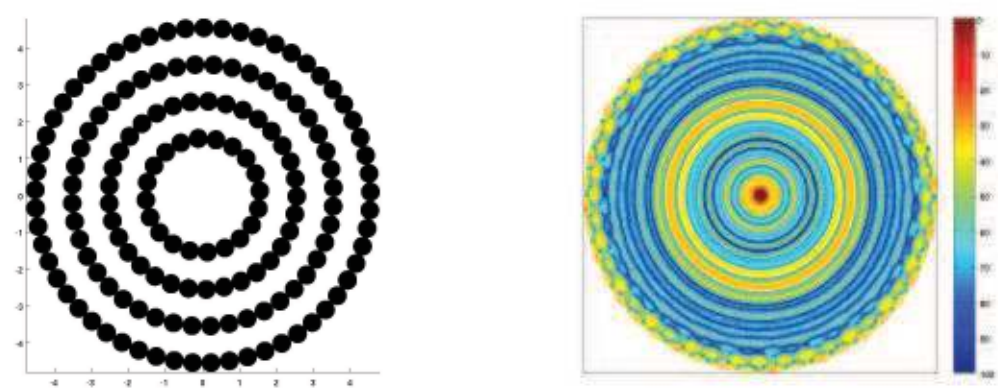

(a) $\mathrm{AR}=.5$
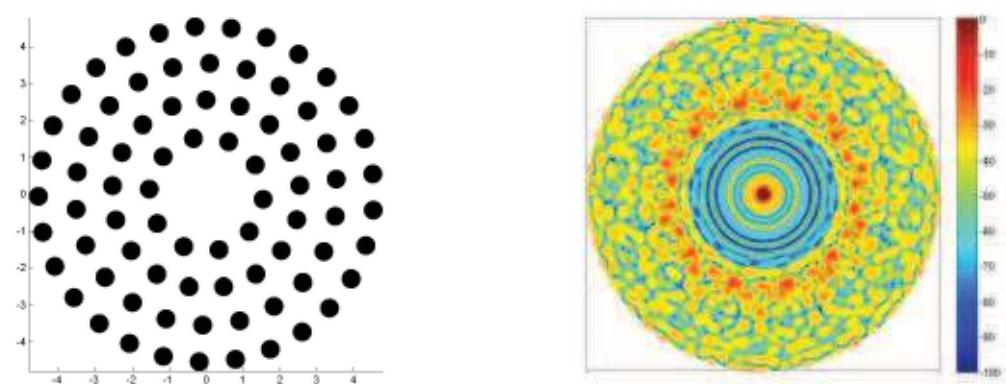

(b) $\mathrm{AR}=1$.
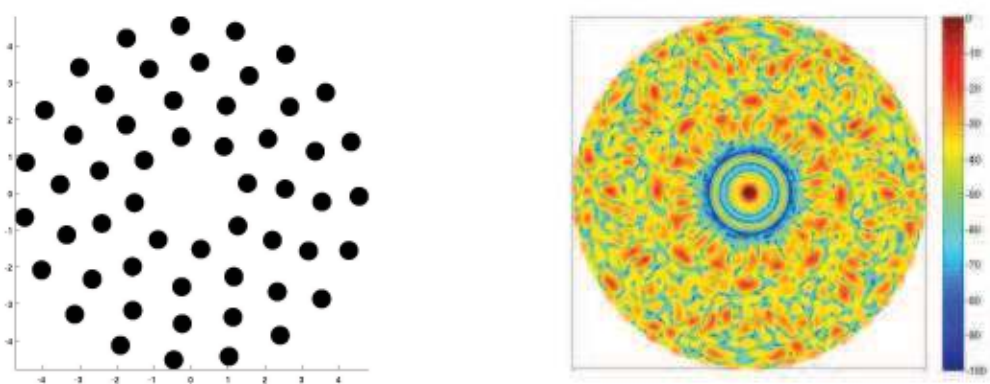

(c) $\mathrm{AR}=1.5$

Fig. 9. Aperture of $d_{R}=2 \lambda$ and $D=20 \lambda$ and Array Factor Lobe distribution with diferent Aspect Ratio.

\subsection{Circular array design procedure}

Circular apertures provide more freedom degrees to improve our design than spiral apertures. The study of Regular Apertures shows that grating lobe distribution can be controlled if radial and angular distances are properly chosen. Futhermore, elements that control grating lobe 


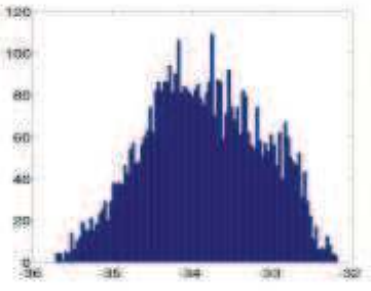

(a) $\mathrm{AR}=0.5$

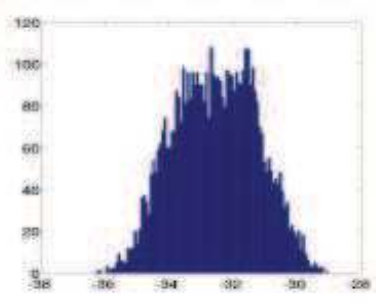

(b) $\mathrm{AR}=1$

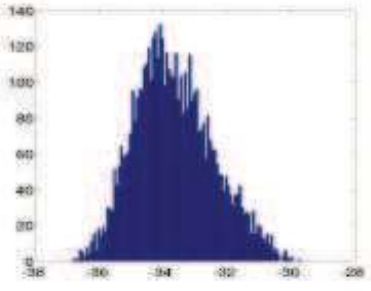

(c) $\mathrm{AR}=1.5$

Fig. 10. Distribution of grating Lobe values for 1000 different apertures wideband (60\%). The three aspect ration are considered.

formation can be independently studied, which allows to divide the design process in several stages.

- Flattening emission beampattern, that is to say:

- Dividing the aperture in equispaced radial regions and avoiding angular grating lobes generation. An optimization process is implemented to spot the rings distribution that produces lower grating lobes.

- With the solution obtained, the aperture is composed with 128 elements. Different interelement distances are used in each ring in order to spread angular lobes over different elevation positions and reduce coincidences. After that, an optimization operation to find the most adequate phases alignment is performed.

- A complementary aperture is obtained. Using the previously obtained solution as a basis and as the emission aperture, the reception aperture for the pulse-echo process is composed by finding the new phases alignment that produces better beampattern

\subsubsection{Reducing radial grating}

At first stage the aperture with $60 \lambda$ diameter and 128 elements is configured using an aspect ratio near to one to obtain a uniform distribution of the elements. A 9 ring configuration is obtained. Figure 10(b) shows how this configuration can produce, in function of its phases alignment, an intial $29 \mathrm{~dB}$ to $36 \mathrm{~dB}$ dynamic range .

Then, the number of elements per ring is increased to eliminate angular grating lobes using $d_{A}=\lambda / 2$. An optimization process is initalized to find the new ring locations that minimize radial grating lobes. To avoid ring concentrations and other non adequate solutions some restrictions were applied to the optimization process. The outer ring has a fixed location to garantee lateral resolution. The other can expand or contract but only within their own regions. The minimun distance between rings is $\lambda$ to allow the use of big elements.

However, we would like to emphasize that since the optimization process was not our objective, only a simple search algoritm was implemented. Figure 11 shows the results, our initial aperture and its lateral profile (minimum, mean and maximum secondary lobes at each elevation position) and the optimized aperture obtained. The grating lobes were initially located at $18^{\circ}$, and after the optimization process they spread over the $10^{\circ}$ to $30^{\circ}$ range. An improvement in the grating lobe level is obtained, it goes down from $-54 \mathrm{~dB}$ to $-61 \mathrm{~dB}$. Table 3 shows the solution obtained. 


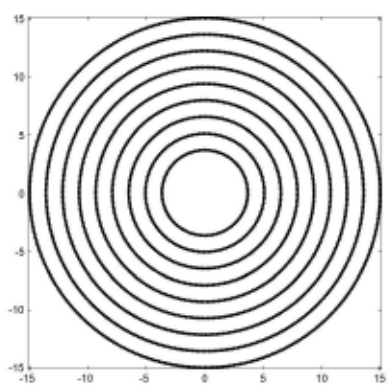

(a) Equispaced-ring distribution

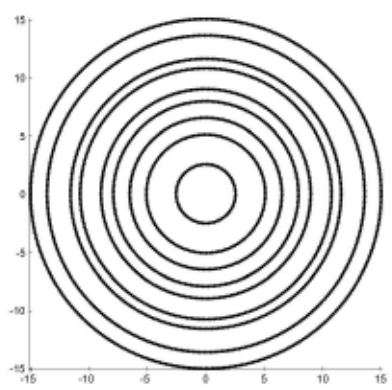

(c) Improved-ring distribution

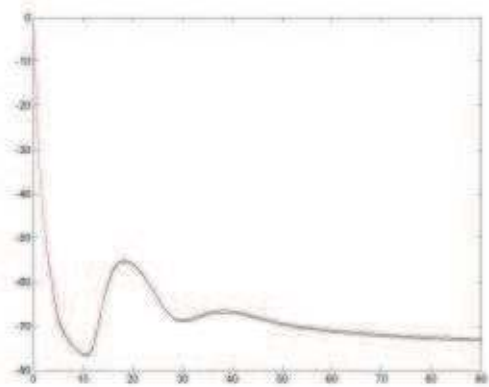

(b) Lateral response in wideband

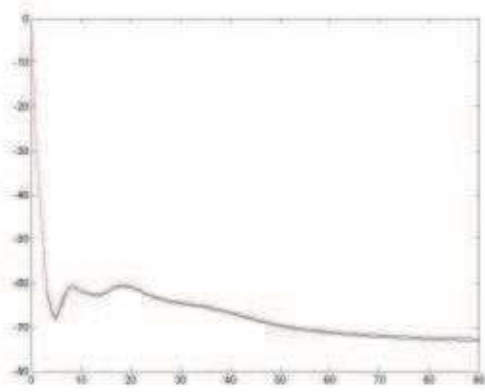

(d) Improved lateral response in wideband

Fig. 11. Improved ring distribution to reduce radial grating lobes

\subsubsection{Reducing angular grating lobes}

To reduce grating angular lobes, the interelement distance varies from one ring to another. Distances increase a $\lambda$ per ring to guarantee the distribution of angular grating lobes over a wide area. However, round operations to discretize the number of elements can cause a change in the estimated garting lobes positions. Obviously, the best solution is to avoid coincidences with the radial grating lobe region. Unfortunately this is not always posible since we are trying to get a wide dispersion. Table 3 shows the configuration and grating lobes positions.

\begin{tabular}{|c|c|c|c|c|c|c|c|c|c|}
\hline RING & 1 & 2 & 3 & 4 & 5 & 6 & 7 & 8 & 9 \\
\hline Radio $(\mathrm{mm})$ & 2.53 & 5.08 & 6.5 & 7.92 & 8.96 & 10.75 & 11.57 & 13.58 & 15 \\
\hline$N^{0}$ elem. & 11 & 13 & 14 & 15 & 14 & 15 & 15 & 16 & 15 \\
\hline$d_{A}(\mathrm{~mm})$ & 1.45 & 2.45 & 2.91 & 3.31 & 4.02 & 4.5 & 4.84 & 5.33 & 6.28 \\
\hline$G L_{A} \cdot(\mathrm{deg})$ & 20.16 & 11.73 & 9.86 & 8.6 & 7.13 & 6.37 & 5.92 & 5.37 & 4.56 \\
\hline
\end{tabular}

Table 3. Element configuration of the optimized aperture.

The dynamic range obtained goes up $36 \mathrm{~dB}$. Then, an optimization algorithm is implemented to search a better phase alignement and thus improve the result. The new dynamic range goes up $40 \mathrm{~dB}$, that is to say a $4 \mathrm{~dB}$ gain. 


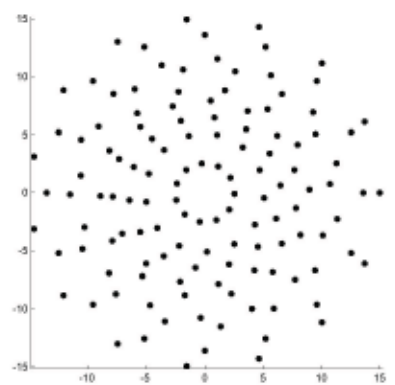

(a) Element distribution

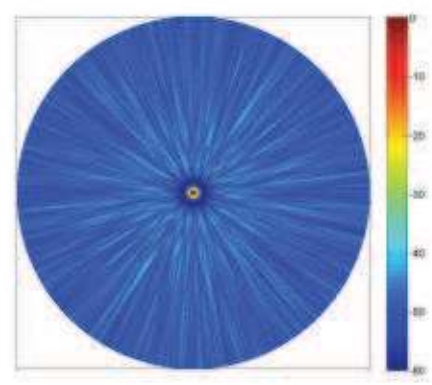

(b) Beampattern in the semisphere

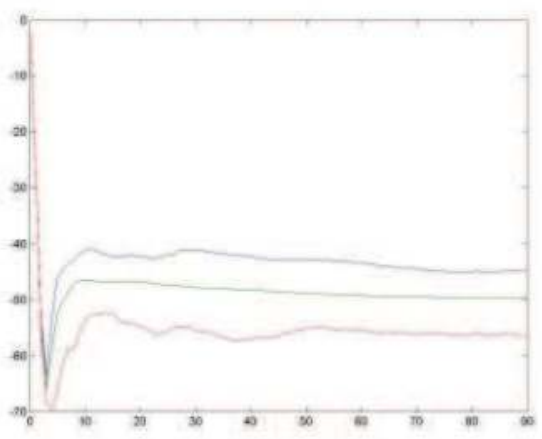

(c) Lateral profile. Maximum, mean and minimum side lobes in elevation are presented

Fig. 12. Best result for $\mathrm{CA}(128)$ is presented.

\subsubsection{Complementary aperture}

Finally, we need to find the complementary aperture that reduces the grating lobe in the pulse-echo response. With this aim, the $\mathrm{CA}(128)$ is used as reference and emission aperture and a new phase alignment is searched for the reception configuration. Figure 13 shows the solution obtained.

Table 4 shows $\mathrm{CA}(128)$ and $\mathrm{CA}(128,128)$ parameters. The dynamic range is improved by $4 \mathrm{~dB}$ and the MSR is significantly better when complementary apertures are used. Both apertures present equal lateral resolution.

\begin{tabular}{|c|c|c|cc|c|}
\hline & & & \multicolumn{2}{|c|}{ Lat. Res. } & MSR \\
\hline Configuration & $\mathrm{D}$ & GL Level $(60 \%)$ & $-6 \mathrm{~dB}$ & $-40 \mathrm{~dB}$ & $-40 \mathrm{~dB}$ \\
\hline $\mathrm{CA}(128)$ & $60 \lambda$ & $-40.5 \mathrm{~dB}$ & $1.0^{\circ}$ & $2.7^{\circ}$ & $10.8 \mathrm{~dB}$ \\
\hline $\mathrm{CA}(128,128)$ & $60 \lambda$ & $-44.5 \mathrm{~dB}$ & $1.0^{\circ}$ & $2.7^{\circ}$ & $17.26 \mathrm{~dB}$ \\
\hline
\end{tabular}

Table 4. Performance of the best CA(128) configuration and the CA(128,128). 


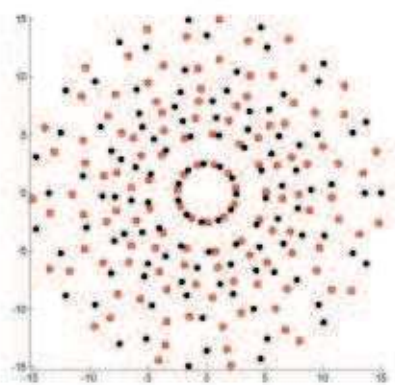

(a) Element distribution: in black emission aperture; in red reception aperture

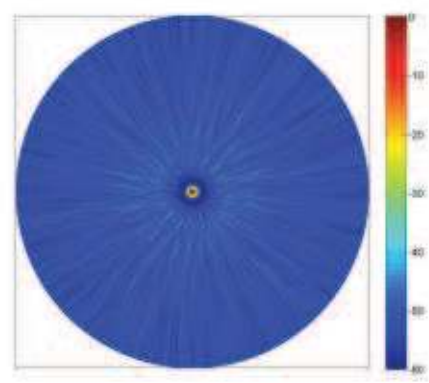

(b) Beampattern in the semisphere

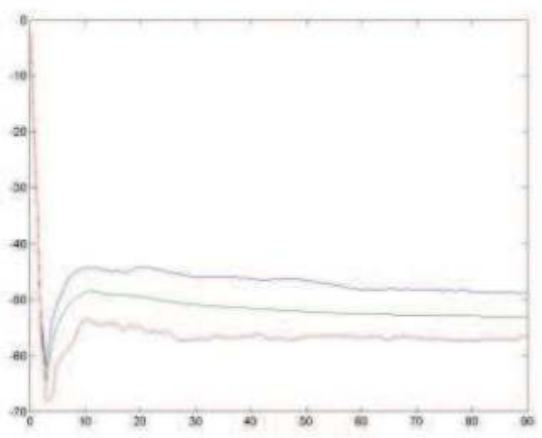

(c) Lateral profile. Maximum, mean and minimum side lobes in elevation are presented

Fig. 13. Best result for $\mathrm{CA}(128,128)$ is presented.

\section{Conclusions}

As this paper shows, both the Fermat Spiral and the Circular Apertures are interesting distributions for designing bidimensional sparse arrays. The mechanisms that determine the grating lobes formation were studied. For each configuration, two design strategies based on the multiplicative effect of the emission reception response were studied.

Both apertures produce similar results. Spiral apertures do slightly better in dynamic range whereas circular apertures, in turn, in lateral resolution. Both present adequate values to be used in some Non Destructive Testing applications, that is a dynamic range of near $45 \mathrm{~dB}$ and $2.5^{\circ}$ of lateral resolution at $(-40 \mathrm{~dB})$. However the strategies used to obtain the best solutions were different.

- In the Fermat Spiral design, since there are no constructive parameters we need to find the most adequate solutions by exhaustive computation in one of the identified regions. In this sense SP1, SP2 and SP3 regions offer good solutions with similar results. The main difference between them is the number of branches that organize element distribution. 
Some solutions can be more easily implemented than others. Choosing the adequate solution could simplify the manufacturing process.

- In the Circular Apertures case, the solution is given by an optimization process. The elements that generate grating lobes were analysed. Taking into account this analysis the process was divided in several stages. At each stage, a particular constructive parameter was isolated and optimized. Although the optimization process was very simple, the procedure was able to improve the initial aperture by $10 \mathrm{~dB}$. Therefore we could assume that better results could be obtained if more sofisticated optimization process were employed.

To compare our results with other configurations we have used the existing examples in literature. Sumanaweera at Sumanaweera et al (1999) presents several examples for configurations with a $40 \lambda$ diameter: a Archimedean Spiral SP $(128,127)$ a random M $(192,64)$ and a periodic $\mathrm{M}(128,127)$ with dynamic ranges of $40 \mathrm{~dB}, 30 \mathrm{~dB}$ and $15 \mathrm{~dB}$ respectively. Nivolov at Nikolov \& Jensen (2000) studied $18 \lambda$ apertures using different arragements based on matrix aperture and the convolution far field model of the pulse-eco in the complementary apertures design. He obtaned a dynamic range of $49 \mathrm{~dB}$ with a $\mathrm{M}(256,256)$ configuration. Also, on the same basis, Austeng at Austeng \& Holm (2002) studies different matrix configurations for $25 \lambda$. He extends the Vernier models in directions other than the main axes (radial, diagonal) and compares them with random configurations with different sparsing distribution functions (binned, polar). Although Austeng uses more elements than we do, we can compare our results with smaller apertures that achieve a dynamic range of: $39.9 \mathrm{~dB}$ for Vernier $\mathrm{M}(421,208)$, $48.5 \mathrm{~dB}$ for polar random distribution $\mathrm{M}(484,361)$ and 51.6 for binned random distribution $\mathrm{M}(447,447)$. More recently, Oliveri has presented a non-overlamping solution $\mathrm{M}(265,264)$ with a dynamic range of $40 \mathrm{~dB}$.

In general our results are better than those found in the literature. Although our dynamic range goes up to $45 \mathrm{~dB}$, we obtain a better lateral resolution (our aperture is bigger) with a number of elements that is significantly lower. Futhermore, when the number of elements is comparable, our apertures provide better dynamic range.

\section{Acknowledgments}

This study was partly supported by the Spanish Ministry of Science and Innovation under projects DPI2007- 65408-C02-01, DPI2007-65408-C02-02, and PSE-020000-2009-10 (PSS-020000-2009-25 subproject) which is co-supported by the ERDF (European Regional Development Fund).

\section{References}

S.W. Smith, H.G. Pavy and O.T. von Ramm (1991) High-speed ultrasound volumetric imaging system, Part I: Transducer design and beam steering. IEEE Tans. on UFFC. vol 38(2). pp. 101-108. ISSN: 0885-3010.

W.J Hendricks (1991) Totally random versus the bin approach for random arrays. IEEE Trans on Antennas and Propagation. vol 39(12). pp.1757-1761. ISSN: 0018-926X.

J.L. Schwartz and B.D. Steinberg, (1998) Ultrasparse, ultrawideband arrays. IEEE Tans. on UFFC. vol 45(2). pp. 376-393. ISSN: 0885-3010.

G.R. Lockwood and F.S. Foster, (1996) Optimizing the radiation pattern od sparse periodic two-dimensional arrays. IEEE Tans. on UFFC. vol 45(2). pp. 338-348. ISSN: 0885-3010. 
A. Austeng and Sverre Holm. (2002) Sparse 2D arrays for 3D Phased Array Imaging Design Methods. IEEE Tans. on UFFC. vol 49(8). pp. 1073-1086. ISSN: 0885-3010.

S.I. Nikolov and J.A. Jensen (2000) Application of different spatial sampling patterns for sparse array transducer design. Ultrasonics. vol 37. pp. 667-671. ISSN: 0041-624X.

Y. Mendelsohn and E. Wiener-Avnear (2002) Simulations of circular 2D phase-array ultrasonic imaging transducers. Ultrasonics. vol. 39 (9). pp. 657-666. ISSN: 0041-624X.

Ullate LG, Godoy G and Martinez O. (2006) Beam steering with segmented annular arrays. IEEE Tans. on UFFC. vol 53 (10). pp. 1944-1954. ISSN: 0885-3010.

V. Bavaro, G. Caliano and M. Pappalardo. (2008) Element Shape Designo of 2D CMUT Arrays for Reducing Grating Lobes. IEEE Tans. on UFFC. vol 55(2). pp.308-318. ISSN: 0885-3010.

T.S. Sumanaweera, J. Schwartz and D. Napolitano (1999) A spiral 2D phased array for 3D imaging. 1999 IEEE Ultrasonics Symposium. Proceedings. pp.1271-1274.

O. Martínez-Graullera, C.J. Martín, Gregorio Godoy, Luis Gomez-Ullate (2010) 2D Array design based on Fermat Spiral for ultrasound imaging. Ultrasonics. vol. 50 (2). pp. 280-289. ISSN: 0041-624X.

B. Piwakowski and K. Sbai,(1999) A new approach to calculate the field radiated from arbitrarily structured transducer arrays, IEEE Trans. Ultrason., Ferroelect., Freq. Contr., vol. 46, n. 2, pp. 422-439. ISSN: 0885-3010.

Roger V. Jean, (1983) Mathematical Modeling in Phyllotaxis: The State of the Art. Mathematical Biosciences. vol 64. pp. 1-27. ISSN: 0025-5564.

J.N. Ridley. (1982) Packing Efficiency in Sunflower Heads. Mathematical Biosciences. vol 58. pp. 129-39. ISSN: 0025-5564

Giacomo Oliveri and Andrea Massa,(2010) ADS-based array design for 2-D and 3-D ultrasound imaging.. IEEE Tans. on UFFC. vol 57(7). pp. 1568-82. ISSN: 0885-3010. 


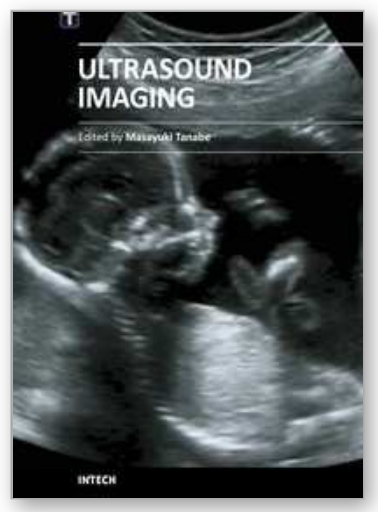

\author{
Ultrasound Imaging \\ Edited by Mr Masayuki Tanabe
}

ISBN 978-953-307-239-5

Hard cover, 210 pages

Publisher InTech

Published online 11, April, 2011

Published in print edition April, 2011

In this book, we present a dozen state of the art developments for ultrasound imaging, for example, hardware implementation, transducer, beamforming, signal processing, measurement of elasticity and diagnosis. The editors would like to thank all the chapter authors, who focused on the publication of this book.

\title{
How to reference
}

In order to correctly reference this scholarly work, feel free to copy and paste the following:

Oscar Martinez-Graullera, Luis Gomez-Ullate, David Romero, Carlos J. Martin and Gregorio Godoy (2011). Design of Curvilinear Array Apertures for 3D Ultrasonic Imaging, Ultrasound Imaging, Mr Masayuki Tanabe (Ed.), ISBN: 978-953-307-239-5, InTech, Available from: http://www.intechopen.com/books/ultrasoundimaging/design-of-curvilinear-array-apertures-for-3d-ultrasonic-imaging

\section{INTECH}

open science | open minds

\section{InTech Europe}

University Campus STeP Ri

Slavka Krautzeka 83/A

51000 Rijeka, Croatia

Phone: +385 (51) 770447

Fax: +385 (51) 686166

www.intechopen.com

\section{InTech China}

Unit 405, Office Block, Hotel Equatorial Shanghai

No.65, Yan An Road (West), Shanghai, 200040, China 中国上海市延安西路65号上海国际贵都大饭店办公楼 405 单元

Phone: +86-21-62489820

Fax: $+86-21-62489821$ 
(C) 2011 The Author(s). Licensee IntechOpen. This chapter is distributed under the terms of the Creative Commons Attribution-NonCommercialShareAlike-3.0 License, which permits use, distribution and reproduction for non-commercial purposes, provided the original is properly cited and derivative works building on this content are distributed under the same license. 